\title{
Préparer les étudiants en médecine à affronter l'influence de l'industrie pharmaceutique : un enjeu éthique, professionnel et éducationnel
}

\section{Preparing medical students to address pharmaceutical industry influence: an ethical, professional and educational issue}

\section{Paul SCHEFFER ${ }^{1}$}

1 Doctorant en sciences de l'éducation (Université de Paris 8, Equipe d'accueil Centre de recherche interuniversitaire, expérience, ressources culturelles, éducation -EXPERICE), administrateur du Formindep.

Manuscrit soumis à la rédaction le 6 juillet 2016 ; commentaires éditoriaux formulés à l'auteur le 8 et le 16 novembre 2016 ; accepté pour publication le 4 décembre 2016

\author{
Mots-clés \\ indépendance ; \\ industrie \\ pharmaceutique ; \\ American Medical \\ Student Association ; \\ Association Nationale \\ des Étudiants en \\ Médecine de France ; \\ professionnalisme ; \\ éthique ; conflits \\ d'intérêts
}

\section{Keywords}

Independence;

Pharmaceutical

industry; American

Medical Student
Résumé - Contexte : L'influence systémique de l'industrie pharmaceutique en médecine est étudiée depuis des décennies. La recherche et la clinique sont fortement touchées, avec des enjeux sanitaires, économiques et éthiques majeurs. Exégèse : La faiblesse des études médicales, en termes de formation à l'indépendance, est régulièrement identifiée comme l'un des secteurs prioritaires où des changements conséquents sont à apporter. Ceci serait d'autant plus nécessaire que les étudiants sont eux-mêmes soumis à l'influence des firmes tout en se croyant immunisés face à cette dernière. Cet article présente les réponses apportées à ce jour au niveau international, que cela soit les politiques officielles adoptées par certaines facultés vis-à-vis des conflits d'intérêts, bien souvent sous la pression des étudiants, ou l'évaluation des dispositifs pédagogiques expérimentés. Une proposition de curriculum global (The Pharmfree curriculum) a également été élaborée par l'American Medical Student Association (AMSA). Conclusion : Dans l'espace francophone, des initiatives ont été identifiées au Canada et en France qui pourraient servir aux acteurs des autres pays de cette zone souhaitant agir dans ce domaine.

Abstract - Background: The systemic influence of the pharmaceutical industry in medicine has been studied for decades. The medical research and clinical fields are profoundly impacted, with major health, economical and ethical consequences at stake. Analysis: The weakness of medical studies in terms of independence training is regularly identified as one of the priority sectors where important changes need to be made. It would all the more crucial as 


\author{
Association; \\ Association nationale \\ des étudiants en \\ médecine de France; \\ Professionalism; \\ Ethics; \\ Conflict of interests
}

students themselves are targeted by the firms' influence and believe that it does not affect them. The article offers answers provided so far at the international level, whether on conflicts of interest policies adopted by different medical schools, often pressured by medical students, or the evaluation of pedagogical experiences carried out. The American Medical Student Association also suggested a global curriculum - The Pharmfree Curriculum. Conclusion: In French-speaking regions, Canada and France, some initiatives were identified and could be used by other countries wishing to take action in this subject.

\section{Introduction}

L'influence de l'industrie pharmaceutique en médecine reste l'un des défis majeurs de la médecine moderne, comme le soutient notamment le professeur de droit Marc Rodwin ${ }^{[1]}$, ainsi que de nombreux observateurs $^{[1]}$. Cependant, même si cette problématique fait l'objet de nombreux travaux, il est pourtant moins connu que cette influence concerne également la formation initiale des médecins. Cet article souhaite revenir sur ce point et présenter un état des lieux des réponses apportées à cette situation au niveau international, en particulier dans l'espace francophone.

\section{Problématique}

Dans un article intitulé « Selling Drugs by "Educating" Physicians ? », publié en 1961 dans le Journal of Medical Education ${ }^{[2]}$, le professeur de pédiatrie Charles May décrit déjà l'influence systémique de l'industrie pharmaceutique dans le champ de la santé, qui a depuis fait l'objet de nombreux rapports et travaux venus préciser et confirmer son emprise. L'Institute of Medicine (IOM) aux États-Unis a notamment édité en 2009 un volumineux ouvrage sur les conflits d'intérêts dans la recherche, la formation et le soin en médecine ${ }^{[3]}$. Différentes instances politiques ont également exprimé leur préoccupation à ce sujet, à l'instar du parlement britannique en 2006, dans un rapport dont les conclusions étaient reprises de la manière suivante par un commentateur dans la revue The Lancet :

L'influence de l'industrie pharmaceutique est énorme et hors de tout contrôle.
Ses tentacules s'infiltrent à tous les niveaux, atteignant les patients, les ministères de la Santé, les organes de régulation, les gestionnaires, les chercheurs, et les organisations humanitaires médicales, puis les universitaires, les médias, les aidants, les écoliers, et les responsables politiques. Les grandes multinationales planifient, sponsorisent, orchestrent et contrôlent les publications de tous les essais de médicaments clés ; elles assurent la production, la mise sur le marché, et la promotion des médicaments que nous consommons $^{[4]}$.

De nombreux scandales ont éclaté ces dernières années dans différents pays, comme c'est le cas en France. Du retrait de quatre recommandations de la Haute Autorité de Santé (HAS) entachées de conflits d'intérêts ${ }^{[5]}$ au soupçon de corruption des membres de la commission d'Autorisation de Mise sur le Marché de l'Agence Nationale de Sécurité du Médicament (ANSM) ${ }^{[6]}$, les exemples s'ajoutant à l'affaire du benfluorex (Mediator $®)$, qui a profondément remis en cause le système de santé français depuis $2010^{[7]}$, ne manquent malheureusement pas.

L'absence de formation à l'indépendance face aux stratégies d'influence des firmes durant les études médicales a été mentionnée par plusieurs observateurs comme l'une des causes majeures de ces défaillances. Les étudiants et les médecins baignent en effet dans une culture de connivence (culture of entitlement) avec l'industrie dès les premières années d'étude $^{[8]}$ :

De même, il nous faudrait réfléchir plus sérieusement aux liens entre les étudiants et les entreprises pharmaceutiques. Il n'est pas normal qu'ils soient encore si étroits et nous pourrions envisager de 
modifier la loi pour mieux les encadrer et les limiter. Pourquoi ? On le sait, plus tôt les liens sont établis, plus les étudiants seront soumis à l'influence prégnante des industries pharmaceutiques. $^{[9]}$

Les études existantes sur les représentations des étudiants vis-à-vis de l'industrie pharmaceutiques montrent en tout état de cause que ces derniers s'avèrent perméables aux stratégies des firmes, tout en se croyant immunisés. Ceci a été étudié dans de nombreux pays, et notamment au Canada et en France pour ce qui concerne l'espace francophone ${ }^{[10-11]}$. Cette formation à l'indépendance s'avère d'autant plus nécessaire que les influences sont souvent difficiles à détecter, provenant notamment de sources d'information reconnues comme faisant autorité. Les leaders d'opinion, souvent recrutés parmi les professeurs de médecine les plus influents, jouent un rôle majeur dans les stratégies de vente des firmes ${ }^{[12]}$. A eux seuls ils représentent en moyenne plus de $30 \%$ du budget marketing des industriels ${ }^{[13]}$. La fiabilité des recommandations de bonnes pratiques sur lesquelles la plupart des médecins apprennent à se baser est aussi fortement remise en cause ${ }^{[14]}$.

Outre l'influence portant sur les connaissances scientifiques, les enjeux sont de taille. L'OMS estime que le mésusage du médicament entraîne la mort de 40000 personnes chaque année en France, dont la moitié serait évitable ${ }^{[15]}$. Au niveau mondial, le médicament se positionne comme la troisième cause de mortalité après le cancer et les maladies cardiovasculaires, chiffre défendu par de nombreuses sommités comme Richard Smith, l'ancien rédacteur en chef du British Medical Journal ${ }^{[16]}$. En termes économiques, 10 à 17 milliards d'euros pourraient également être épargnés chaque année en France ${ }^{[17,18]}$, autant d'argent qui pourrait servir à employer davantage de soignants dans les nombreux services surchargés par exemple.

\section{Une réponse éducationnelle à mettre en œuvre}

Sur le plan éthique, il semble assez évident que les étudiants devraient être informés des stratégies d'influence les prenant pour cible, afin de protéger au mieux leurs futurs patients, ne serait-ce que pour être en mesure de respecter les dispositions déontologiques. En France, par exemple, le Code de déontologie souligne le devoir d'indépendance des médecins. L'article 5 pose le fait que « le médecin ne peut aliéner son indépendance professionnelle sous quelque forme que ce soit $»$. L'article 95 précise cet énoncé :

le fait pour un médecin d'être lié dans son exercice professionnel par un contrat ou un statut à une administration, une collectivité ou tout autre organisme public ou privé n'enlève rien à ses devoirs professionnels et en particulier à ses obligations concernant le secret professionnel et l'indépendance de ses décisions. En aucune circonstance, le médecin ne peut accepter de limitation à son indépendance dans son exercice médical de la part de l'entreprise ou de l'organisme qui l'emploie. Il doit toujours agir, en priorité, dans l'intérêt de la santé publique et dans l'intérêt des personnes et de leur sécurité au sein des entreprises ou des collectivités où il exerce. ${ }^{[19]}$

Des dispositions similaires sont formulées dans les textes de certains pays francophones, que cela soit les articles 7, 63 et 73 au Québec ${ }^{[20]}$, les articles 38 et $39 \mathrm{du}$ code suisse ${ }^{[21]}$, ou la position exprimée par les Académies belges de médecine ${ }^{[22]}$.

Il s'agirait en somme que les facultés préparent les futurs médecins à pouvoir déjouer de leur mieux les stratégies d'influence redoutables des firmes, et s'efforcent de protéger les étudiants durant leur formation initiale, ces derniers se retrouvant exposés dès leurs premières années d'étude. En effet, les étudiants rentrent très tôt en contact avec des délégués médicaux envoyés par l'industrie pharmaceutique. Des événements socio-professionnels du type «Journée des internes », sponsorisés par les firmes, peuvent avoir lieu au sein des facultés de médecine. De nombreuses publicités pour des médicaments sont également présentes dans certains manuels. En France, des épreuves classantes nationales « blanches » (sessions d'entraînement aux épreuves classantes nationales officielles de fin de second cycle, organisées à une échelle plus ou moins importante, inter-régionale ou 
nationale) ont été organisées pendant de nombreuses années par certains industriels du médicament. Des séminaires obligatoires de formation dans le cadre des diplômes d'études spécialisés (formation spécialisée post-graduée) sont encore souvent organisés avec le soutien financier de l'industrie pharmaceutique (hébergement et restauration des étudiants). Enfin, et ce n'est pas là une liste exhaustive, les enseignants peuvent avoir des conflits d'intérêts en lien direct avec le contenu de leur enseignement, comme cela a été le cas au Canada $^{[23]}$ et à Harvard ${ }^{[24]}$.

Deux enjeux supplémentaires seraient à prendre en compte. Cette formation à l'indépendance pourrait inciter certains futurs médecins, mieux avertis, à faire le choix de renoncer délibérément à participer aux stratégies de marketing des firmes, et en inciter d'autres à se mobiliser pour agir sur l'environnement institutionnel conditionnant l'exercice de la médecine, à la manière des membres du Formindep, une association qui milite depuis 2004 en France pour une formation et une information indépendantes de tout intérêt autre que celui des patients dans le champ de la santé, ou d'autres organismes agissant dans ce sens.

\section{Des initiatives pionnières et des résultats préliminaires encourageants}

A ce jour, ce besoin de formation manifeste n'est cependant pas encore reconnu et intégré aux programmes d'enseignements par les responsables de formation et les enseignants francophones, à l'exception du Canada plus en avance dans ce domaine. En effet, l'Association des Facultés de Médecine Canadiennes a publié un rapport en 2010, énonçant des mesures auxquelles les facultés de ce pays sont invitées à se conformer, notamment afin de maintenir leur accréditation $^{[25]}$. De profondes carences avaient été constatées lors de l'état des lieux réalisé en $2009^{[11]}$. Des progrès semblent donc avoir été accomplis depuis. Ainsi, en 2015, l'Université Laval, à Québec, a révisé sa politique qui datait de 2009. Des enseignements relatifs à l'influence de l'industrie pharmaceutique ont été introduits dans le premier cycle et dans les programmes de résidence ${ }^{[26]}$. Un enseignant de cette faculté fait également remarquer que dans la mesure du possible, les noms commerciaux des médicaments ne sont pas utilisés dans les cours.

Ces mesures restent cependant toujours fragiles. Le manque de financement public des facultés incite de nombreux enseignants et acteurs à rechercher les contacts avec les firmes, à favoriser une conception positive de ces dernières, considérées comme « partenaires », et à estimer qu'il est préférable pour les étudiants d'avoir des contacts avec les firmes afin d'apprendre à gérer la relation avec elles plutôt que de les éviter ${ }^{[26]}$. Il est à noter que cette position va à l'encontre des recommandations de l'American Medical Student Association (AMSA), qui rappelle que les études sur le sujet montrent que les interactions avec des représentants industriels sont contreproductives en favorisant une conception plus positive des firmes chez les étudiants ${ }^{[27]}$. C'est ce qui a conduit les facultés de médecine norvégiennes, les seules en Europe jusqu'à présent, à convenir d'un accord collectif pour mettre fin aux initiatives en lien avec l'industrie pharmaceutique sur les campus, et exclure cette dernière des activités éducatives. Cet accord date de 2005, nous n'avons pas trouvé d'étude récente rapportant les résultats de ces mesures ${ }^{[28,29]}$.

Les pays francophones pourraient également profiter de l'avancée des États-Unis en la matière, qui ont fait évoluer leur cursus de formation depuis 2008 , suite à la pression des étudiants réunis au sein de leur très active association. L'AMSA a lancé en 2002 la campagne nationale Pharmfree, récemment rebaptisée Just Medicine, visant à réduire autant que possible l'influence des firmes sur la formation initiale. L'AMSA s'est notamment appuyée sur les recommandations du rapport de l'IOM déjà cité et de L'American Association of the Medical Colleges (AAMC), qui réunit les 145 facultés de médecine états-uniennes, les 17 facultés canadiennes accréditées, ainsi que plus de 400 hôpitaux universitaires. L'AAMC s'est en effet également positionnée sur le sujet en publiant en 2008 un rapport où elle défend la nécessité d'intégrer la formation à l'indépendance dans les études médicales ${ }^{[30]}$.

Depuis 2007, l'AMSA réalise chaque année un classement des facultés de médecine états-uniennes 
vis-à-vis de leur politique officielle par rapport aux stratégies d'influence de l'industrie pharmaceutique. Pratiquement aucune faculté ne s'était intéressée à cette question à l'époque, et la grande majorité ont reçu la plus basse note possible. En 2014 cependant, deux-tiers des facultés ont obtenu un $\mathrm{A}$ ou un $\mathrm{B}$, qui correspondent aux meilleurs notes attribuables ${ }^{[31]}$. Il a été montré en outre que les jeunes médecins sortant des facultés s'étant dotés d'un cursus conséquent en matière d'indépendance prescrivaient davantage dans l'intérêt des patients, en étant moins sensibles aux sirènes de l'industrie ${ }^{[32]}$.

Ce résultat a été aussi obtenu grâce à l'implication des étudiants au niveau local comme cela a été rapporté dans un article du New-York Times concernant le cas d'Harvard. En faisant des recherches sur internet, un étudiant s'est rendu compte qu'un de leur enseignant prônait des traitements anti-cholestérol dans ses cours, alors qu'il était consultant pour dix firmes pharmaceutiques, dont cinq les commercialisant. Cette révélation fit scandale, comme l'exprime un étudiant :

Je me suis senti vraiment trahi ; il y a là 160 jeunes gens ouverts d'esprit essayant d'apprendre les bases [de la médecine] dans un espace protégé, et l'information qu'il nous donnait n'était pas aussi objective qu'elle aurait dû l'être selon moi. ${ }^{[24]}$

Ceci a conduit à la mobilisation de plus de 200 étudiants rejoints par quelques enseignants qui, en plus de s'appuyer sur ce cas précis de conflits d'intérêts de leur professeur, ont demandé à leur université de faire des progrès en matière d'indépendance dans la mesure où Harvard avait reçu la note la plus basse du classement de l'AMSA. Harvard est aujourd'hui classée A. Il faut noter que l'AMSA a étendu son classement aux hôpitaux depuis 2014.

L'AMSA a également élaboré une proposition de curriculum global intégrant la question de l'indépendance ${ }^{[27]}$. Cinq champs de compétences ont été définies à cette fin :

- professionnalisme et conflit d'intérêts (COI) ;

- développement des médicaments et des produits de santé ;

- évaluation de la sécurité et de l'efficacité des médicaments et des dispositifs médicaux ;
- marketing et pratique clinique ;

- formation médicale continue (FMC)

Chacun de ces champs de compétences est argumenté et détaillé, ce qui peut constituer une base solide pour concevoir un enseignement dans le domaine. Le curriculum Pharmfree présente aussi les études qui ont évalué les enseignements dans le domaine, et prend parti pour un panaché de dispositifs pédagogiques favorisant l'implication des étudiants (études de scandales sanitaires, analyse critique de documents issus des firmes pharmaceutiques, cours magistraux, lecture critique d'article, recherche bibliographique, discussions en petits groupes de travaux dirigés, débat entre étudiants...). D'une façon générale, les différents dispositifs pédagogiques concernés s'efforcent de s'inscrire dans la perspective du changement du paradigme souvent commenté dans cette revue, en évoluant vers une approche centrée sur l'apprenant. Figurent également une proposition d'intégration concrète de ces dispositifs au curriculum actuel et d'autres considérations, profitant du recul qu'ont pu avoir en la matière les étudiants de l'AMSA, en lien avec les expériences menées dans les facultés états-uniennes.

\section{Un mouvement international à consolider et à fédérer}

Cette dynamique peut clairement servir de modèle à d'autres pays. En France, l'Association Nationale des Étudiants en Médecine de France (ANEMF) s'est approprié le curriculum Pharmfree et certains de ses membres défendent déjà ses idées en commissions facultaires. L'ANEMF a apporté également son soutien officiel ${ }^{[33]}$ au classement des facultés françaises que le Formindep a réalisé en 20152016. Celui-ci devrait être bientôt publié, suivant l'exemple des classements réalisés aux ÉtatsUnis $^{[31]}$, au Canada ${ }^{[11]}$ et en Australie ${ }^{[34]}$. L'ANEMF a aussi mis fin à son partenariat avec la firme pharmaceutique Novartis en 2014, et invite désormais régulièrement à ses congrès des acteurs de l'indépendance dans le champ de la santé comme l'association de formation médicale continue Mieux Prescrire, qui édite en français et en anglais la revue de renommée 
internationale Prescrire, le Formindep, ou le collectif d'étudiants de la Troupe du Rire. Ce dernier a réalisé un livret de 32 pages, tenant dans la blouse des médecins, intitulé « Pourquoi garder son indépendance face aux labos pharmaceutiques ?». Il a reçu le prix Prescrire 2015 et un fort écho médiatique ${ }^{\left[35^{3}\right]}$. Plus de 20000 exemplaires circulent actuellement sur les lieux de formation, et ces étudiants sont sollicités pour intervenir dans différentes facultés. Une vidéo animée en trois minutes présentant le livret et ses enjeux est aussi disponible en ligne ${ }^{[36]}$.

Une forte inertie a été constatée malgré ces initiatives. En France, le thème de l'indépendance est resté parmi les grands absents des trois premières éditions des États généraux de la formation médicale, organisés tous les deux ans par la Conférence des doyens. Pour remédier à cette situation, Pierre Frouard, médecin enseignant membre de l'équipe de Prescrire et moi-même avons organisé la première journée nationale sur la formation à l'indépendance dans les études médicales, le 30 avril 2016. Cet événement visait à rassembler les différents acteurs de la formation initiale, à partager les ressources en matière d'information et d'enseignements, et à envisager une stratégie commune afin de développer la formation dans le domaine. L'ensemble de la journée a été filmé afin de pouvoir en partager les contenus ${ }^{[37]}$.

Plusieurs constats ont pu être faits ou rappelés. Le nouveau président de la Conférence des doyens a apporté son soutien à cette initiative, marquant peutêtre une ouverture plus grande à cette thématique de la part de cette organisation. Outre la forte implication de l'ANEMF, les syndicats d'internes se mobilisent également de plus en plus. Le Syndicat National des Jeunes Médecins Généralistes (SNJMG) porte les valeurs de l'indépendance depuis sa création ; la surprise est venue plutôt de l'Inter-Syndicale Nationale Autonome Représentative des Internes de Médecine Générale (ISNAR-IMG) qui s'implique activement dans le domaine depuis quelques années déjà. $\mathrm{Ne}$ manque plus que l'Inter Syndicat National des Internes (ISNI) dont l'intérêt pour cette question en est encore à ses débuts.

Concernant les enseignants, il a été rappelé que l'OMS avait édité en 2009 un manuel d'enseignement intitulé «Comprendre et répondre à la promotion pharmaceutique». Celui-ci a été traduit et rendu public par la Haute Autorité de Santé (HAS) française en $2013^{[38]}$. Le manuel a reçu le Prix Prescrire cette même année. D'autres ressources peuvent compléter ce manuel. Le Formindep met à disposition différents matériaux à cet effet (articles, vidéos, diaporamas) dans sa rubrique consacrée à la formation initiale ${ }^{[39]}$. Il faut également noter que certains membres du Formindep sont prêts à intervenir dans les cours, tout comme ceux de Prescrire ou de la Troupe du Rire. En parallèle des enseignements en matière $d$ 'indépendance qui devraient être dispensés à la faculté, la journée du 30 avril 2016 a permis de souligner l'importance des expériences qui peuvent être vécues au sein des associations et des collectifs étudiants comme l'ANEMF, la Troupe du Rire et le réseau MEDSI (Mobilisation Étudiante pour le Développement d'une Solidarité Innovante ${ }^{[40]}$. Les enseignants français pourraient encourager leurs étudiants à aller à la rencontre de ces organismes et de leurs actions en cours.

A cette journée manquaient malheureusement les témoignages d'étudiants issus d'autres pays francophones. En revanche, certains étudiants espagnols et italiens sont venus parler de leurs démarches. Il serait bien sûr intéressant de savoir comment les associations nationales francophones ou européennes d'étudiants en médecine d'autres pays agissent ou pourraient réagir à la connaissance des initiatives de leurs homologues états-uniens et français. Des événements ont déjà pu avoir lieu sans que nous ayons pu en prendre connaissance. Si tel était le cas, nous formons le vœu que cet article favorise une meilleure mise en réseau par delà les frontières. Quoi qu'il en soit, les enseignants francophones pourraient interpeller, chacun dans leur pays, les organisations étudiantes, qui ont montré qu'elles avaient un rôle clé à jouer en matière d'indépendance.

L'importance primordiale des modèles de rôle dans la formation des étudiants est aujourd'hui établie $^{[41]}$. Différentes ressources existent pour sensibiliser les étudiants dans ce sens, à défaut de pouvoir réaliser des stages avec des médecins mettant l'indépendance à l'œuvre dans leur pratique. L'article « Docteur Visiteur médical » reprend notamment la trajectoire du psychiatre états-unien Daniel Carlat. Ce 
dernier y analyse comment il a pu être enrôlé par une firme pharmaceutique, malgré son sens critique, avant d'en sortir et de lutter aujourd'hui contre son influence ${ }^{[42]}$. L'article « La résistance des soignants », publié par le docteur Philippe Foucras dans la revue canadienne Éthique Publique, ainsi que le travail de l'anthropologue Anne Véga sur les différents profils de médecins généralistes établis en fonction de leurs habitudes de prescription, pourraient également être mobilisés $^{[43,44]}$. Lors de la journée du 30 avril 2016, une maître de stage a apporté son témoignage pour décrire comment il était possible d'intégrer et de partager ce souci de l'indépendance dans ce contexte de formation $^{[37]}$. Différents témoignages d'étudiants sont également disponibles ${ }^{[45,46]}$.

\section{Conclusion}

La formation des étudiants en médecine à l'indépendance, ainsi que la mise en œuvre d'une formation elle-même la plus indépendante possible sont deux enjeux de taille pour les équipes enseignantes et décisionnelles des facultés de médecine. Pour le moment, ce sont surtout les étudiants qui sont arrivés à faire en sorte que des mesures soient entreprises, montrant un intérêt fort pour cette question. L'exemple des ÉtatsUnis apporte un modèle et des ressources documentaires pouvant inspirer des initiatives similaires dans les autres pays, comme c'est le cas au Canada et en France pour ce qui concerne l'espace francophone. Les équipes enseignantes et encadrantes des facultés de médecine ont elles aussi un rôle certain à jouer dans cette évolution des programmes et des pratiques institutionnelles. Puisse cet article contribuer à aider celles et ceux d'entre eux qui décideront d'avancer dans ce sens.

\section{Déclaration d'intérêts}

L'auteur ne déclare aucun lien d'intérêt avec l'industrie pharmaceutique et celle des produits de santé. Il est administrateur du Formindep et membre de La Troupe du Rire.

\section{Approbation éthique}

\author{
Sans objet
}

\section{Références}

1. Rodwin MA. Les conflits d'intérêts en médecine France, Etats-Unis, Japon. Rennes : Presses de l'EHESP, 2014

2. May CD. Selling Drugs by "Educating" Physicians? J Med Educ 1961;3:1-23

3. Lo B, Field MJ (Eds.). Conflict of Interest in Medical Research, Education and Practice. Washington : The National Academies Press, 2009

4. Collier J. Big pharma and the UK Government. The Lancet 2006;367:97-8.

5. Delarue L-A. Une thèse de médecine à l'épreuve d'une formation médicale sous influence. In : Scheffer Paul (Ed.) Les métiers de la santé face aux industries pharmaceutique, agroalimentaire, et chimique : quelles formations critiques ? Paris : L'Harmattan, 2015:105-116

6. Hajdenberg M, Pascariello P. Les gendarmes du médicament faisaient affaire avec les labos. Mediapart. 24 Mars 2015 [On-line]. Disponible sur : https:// www.mediapart.fr/journal/france/240315/les-gendarmes-du-medicament-faisaient-affaire-avec-leslaboratoires?onglet=full

7. Mullard A. Mediator scandal rocks French medical community. Lancet 2011;377:890-892.

8. Brody H. Hooked - Ethics, the Medical Profession, and the Pharmaceutical Industry. Lanham : Rowman \& Littlefield Publishers, 2008

9. Lemorton C, Robinet A. Rapport d'information sur la mise en œuvre de la loi du 29 décembre 2011 relative au renforcement de la sécurité sanitaire du médicament et des produits de santé, Assemblée Nationale, 2013 [On-line]. Disponible sur : http://www.assemblee-nationale.fr/14/rap-info/i1281.asp

10. Etain B, Guittet L, Weiss N, Gajdos V, Katsahian S. Attitudes of Medical Students towards Conflict of Interest : A National Survey in France. PLoS Medicine 2014;9:e92858 [On-line]. Disponible sur : http:/ /journals.plos.org/plosone/article?id=10.1371/journal.pone. 0092858 
11. Shnier A, Lexchin J, Mintzes B, Jutel A-M, Holloway K. Too Few, Too Weak: Conflict of Interest Policies at Canadian Medical Schools. PLoS ONE 2013;8:e68633. [On-line]. Disponible sur : http:// journals.plos.org/plosone/article?id=10.1371/journal.pone.0068633

12. Bras P-L, Ricordeau P, Roussille B, Saintoyant V. L'information des médecins généralistes sur le médicament, Rapport IGAS n ${ }^{\circ}$ RM 2007-136P, 2007 [Online]. Disponible sur : http://www.ladocumentationfrancaise.fr/var/storage/rapports-publics/ 074000703.pdf

13. La Revue Prescrire. Leaders d'opinion : coûteux, mais rentables pour les firmes. Prescrire 2005;266:777

14. Lenzer J. Why we can't trust clinical guideline. BMJ 2013;346:f3830. [On-line]. Disponible sur : http:// www.bmj.com/content/346/bmj.f3830

15. APM International. Mortalité liée aux erreurs médicales : le Lien dénonce "l'omerta" française. 20 janvier 2015.

16. Smith R. Foreword. In : Gøtzsche P., Deadly Medicines and Organised Crime, London : Radcliffe Publishing, 2013:IX

17. Santi P. Michèle Rivasi appelle à une opération mains propres dans la santé. Le Monde, 6 janvier 2015.

18. De Kervasdoué J. Comptes rendus de la mission d'évaluation et de contrôle de la sécurité sociale. Sénat. 2012 [On-line]. Disponible sur : http:// www.senat.fr/compte-rendu-commissions/ 20120213/mecss.html

19. Conseil National de l'Ordre des Médecins. Code de déontologie médicale. CNOM, 2016 [On-line]. Disponible sur : https://www.conseilnational.medecin.fr/sites/default/files/codedeont.pdf

20. Collège des médecins du Québec. Code de déontologie des médecins. 2015 [On-line]. Disponible sur : http://www.cmq.org/publications-pdf/p-6-2015-0107 -fr-code-de-deontologie-desmedecins.pdf? $\mathrm{t}=1479745136689$

21. Fédération des médecins suisses. Code de déontologie de la FMH. 2016 [On-line]. Disponible sur : http:// www.fmh.ch/files/pdf18/

Standesordnung_August_2016_F.pdf

22. Académie royale de médecine en Belgique. Relations entre médecins et entreprises en Belgique. 28 sep- tembre 2002 [On-line]. Disponible sur : http:// www.armb.be/index.php?id=1559

23. Persaud N. Questionable content of an industry-supported medical school lecture series : a case study. Journal of Medical Ethics. 2013 [On-line]. Disponible sur : http://jme.bmj.com/content/early/2013/ 06/10/medethics-2013-101343.abstract

24. Wilson D. Harvard Medical School in Ethics Quandary. New York Times, 2 mars 2009

25. Association of Faculties of Medicine of Canada (AFMC). Position Paper on the Role of Industry in University-Based CME/CPD. 2010 [On-line]. Disponible sur : https://www.afmc.ca/pdf/committees/ SCCPD_Position_Paper_on_Role_of_Industry_in_ University_Based_CME-CPD_Dec\%201_2010.pdf

26. Direction de l'imputabilité sociale et du professionnalisme. Politique en matière de conflits d'intérêts dans les activités de formation, d'évaluation et de recherche. Université Laval, Faculté de médecine, Canada. 2015 [On-line]. Disponible sur : http:// www.fmed.ulaval.ca/fileadmin/documents/facultereseau/documents-officiels-et-promotionnels/documents/politique-conflits-interets-activites-formation-recherche.pdf

27. American Medical Students Association. Evidence and Recommendations for a Model PharmFree Curriculum. 2015 AMSA. [On-line]. Disponible sur : http://www.amsa.org/wp-content/uploads/2015/03/ ModelPharmFreeCurriculum.pdf

28. Lea D, Spigset O, Slørdal L. Norwegian medical students' attitudes towards the pharmaceutical industry. Eur J Clin Pharmacol 2010;66:727-33

29. American Association of Medical Colleges. Industry Funding of Medical Education: Report of an AAMC Task Force. AAMC, 2008 [On-line]. Disponible sur : https://members.aamc.org/eweb/upload/Industry\%20Funding\%20of\%20Medical\%20Education.p df

30. Ce paragraphe est une adaptation d'un passage de l'article suivant : Scheffer P, Guy-Coichard C, OuthGauer D, Calet-Froissart Z, Boursier M, Mintzes B. Borde JS, Confict of Interst Policies at French Medical Schools : Starting from the Bottom, PLOS ONE, 2016 [On line]. Disponible sur : http://dx.plos.org/ 10.1371/journal.pone.0168258 
31. American Medical Students Association. Conflicts of interest policies at medical schools. AMSA Scorecard. 2014 [On-line]. Disponible sur : http://amsascorecard.wpengine.com/

32. King M, Essick C, Bearman P, Cole J, Ross JS. Medical school gift restriction policies and physician prescribing of newly marketed psychotropic medications. BMJ 2013;346:f264

33. Association Nationale des Etudiants en Médecine de France. Lancement d'un état des lieux sur l'indépendance de la formation médicale. 2015 [Online]. Disponible sur : http://www.formindep.org/ IMG/pdf/lettre_de_1_anemf.pdf

34. Mason PR, Tattersall MHN. Conflicts of interest: a review of institutional policy in Australian medical schools. MJA 2011;94:121-125

35. La Troupe du Rire. Pourquoi garder son indépendance face aux labos pharmaceutiques? 2015 [On-line]. Disponible sur : https://latroupedurire.fr/2016/04/ 13/le-livret/

36. La Troupe du Rire. Pourquoi garder son indépendance face à l'industrie pharmaceutique? Vidéo de la Troupe du Rire. 2015 [On-line]. Disponible sur : https://www.youtube.com/watch?v=j5vB15DWbh8

37. Journée d'étude nationale sur la formation à l'indépendance dans les études médicales. Paris. 30 avril 2016 [On-line]. Disponible sur : https:// www.youtube.com/playlist?list=PL5vzBp9poqMUI75wGcKZ02viXgE4Olg Dv

38. Organisation Mondiale de la Santé et Action Internationale pour la Santé. Projet collaboratif. Connaître la promotion pharmaceutique et y répondre. OMS/ HAI, 2013 [On-line] Disponible sur : http:// www.has-sante.fr/portail/upload/docs/application/ pdf/2013-04/

comprendre_la_promotion_pharmaceutique_et_y_r epondre_-_un_manuel_pratique.pdf
39. Formindep. Agir pour l'indépenance. 2016 [On-line]. Disponible sur : http://www.formindep.org/Agirpour-l-independance-en-sante.html

40. Mobilisation internationale pour le développement d'une solidarité internationale (MEDSI). http:// www.medsi.fr/

41. Chamberland M, Hivon R. Les compétences de l'enseignant clinician et le modèle de rôle en formation clinique. Pédagogie Médicale 2005;6:88-111.

42. Carlat D. Docteur Visiteur Médical. New-York Times, 25 novembre 2007. Article traduit par le Formindep [On-line]. Disponible sur : http://www.formindep.org/IMG/pdf/DrVM-NYT.pdf

43. Foucras P. La Résistance des soignants. Éthique publique, 2006;2:18-25. [On-line]. Disponible sur : http://www.adnc.asso.fr/Articles-publies-par-despersonnes

44. Véga A. Le partage des responsabilités en médecine. Une approche socio-anthropologique des pratiques soignantes - Cuisine et dépendance : les usages socioculturels du médicament chez les médecins généralistes français, Rapport remis à la CNAMTS, 2011 [On-line]. Disponible sur : http://www.formindep.org/IMG/pdf/rapport_final3.pdf

45. Témoignages filmés d'étudiants en médecine par rapport à l'influence de l'industrie pharmaceutique pendant leurs études. Prix Prescrire, Paris 2015 [Online]. Disponible sur : https://www.youtube.com/ watch?v=AnIbGhTXjBo

46. Calet-Froissart Z. Comment concilier éducation populaire et formation médicale ? In : Paul Scheffer (Ed.) Les métiers de la santé face aux industries pharmaceutique, agroalimentaire, et chimique : quelles formations critiques ? Paris: L'Harmattan, 2015:8198

Correspondance et offprints: Paul Scheffer, 24 rue des vieilles prisons, 26400 Crest, France.

Mailto : scheffer2003@hotmail.com 